\title{
Umweltpolitik in neuem Rahmen
}

\author{
Die doppelte Globalisierung von Wirtschaftsbeziehungen und zentralen Umwelt- \\ problemen stellt neve Anforderungen an eine erfolgreiche Umweltpolitik. Den- \\ noch bleibt auch für nationale Politiken noch Spielraum.
}

G halisierung räumliche Ausdehnung von Märkten und deren Vernetzung, ist ein wesentliches Element der gegenwärtigen wirtschaftlichen Entwicklung. Umweltrelevant ist sie in zweierlei Hinsicht. Zum einen erschwert die mit der Globalisierung der Wirtschaftsbeziehungen verbundene Intensivierung des Wettbewerbs eine nationale Umweltpolitik: Sie ist (oder scheint zumindest) im Wettbewerb der Systeme gefährdet. Zweitens aber hat die Umweltpolitik selbst eine globale Dimension angenommen: Die zentralen umweltpolitischen Probleme, wie z.B. das 'Ozonloch' oder die 'Klimakatastrophe', sind nicht mehr nationaler, sondern globaler Natur. Ihnen kann deshalb nur mit Hilfe einer international koordinierten Politik begegnet werden.

Für die Globalisierung der Umweltprobleme gibt es (mindestens) drei Ursachen. Zum einen hat die Internationalisierung zu einem erheblichen Anwachsen der Handelsströme geführt, die von einem entsprechenden Anwachsen umweltbelastender Transportleistungen begleitet wird. Als zweites wäre ganz allgemein das Anwachsen der mit der Produktion verbundenen Belastung der natürlichen Umwelt zu nennen. Um die begrenzte Aufnahmefähigkeit der natïrlichen Umwelt für Schadstoffe (scheinbar) zu überspielen, haben wir uns weitgehend zur 'Politik des hohen Schornsteins' entschlossen. Da die meisten Staaten so handeln, exportieren alle Staaten nicht nur Luftschadstoffe, sondern sie importieren sie auch, und die Gesamtbelastung ist dadurch höher, als nach streng volkswirtschaftlichem Kalkül optimal wäre. Entsprechendes gilt auch für andere Schadstoffe. Die eigentliche Globalisierung der Umweltproblematik entstand jedoch dadurch, daß im Zuge der wirtschaftlichen Entwicklung verstärkt auch solche Schadstoffe emittiert wurden, deren globale Wirkung sich unabhängig davon entfaltet, wo sie emittiert werden. Dies gilt z.B. fuir all jene Schadstoffe, welche die Ozonschicht angreifen, insbesondere für die fluorierten Kohlenwasserstoffe (FCKW), aber auch für jene Emissionen, die zur globalen Erwärmung beitragen, vor allem das Kohlendioxid.

Es ist offensichtlich, daß diese globalen Umweltprobleme nur dann wirklich bewältigt werden können, wenn es gelingt, international koordinierte Strategien zu erarbeiten, zu beschließen und durchzusetzen (1). Ein einzelnes Land kann allein keinen wesentlichen Beitrag zur globalen Reduktion dieser Emissionen leisten. Es kann allenfalls eine Vorreiterfunktion einnehmen (2).

\section{Probleme internationaler Kooperation}

Auf der internationalen Ebene fehlt jedoch jene übergeordnete Instanz, die, wie auf nationaler Ebene der Staat, Umweltschutzbestimmungen erlassen und durchsetzen kann. Vielmehr sind wir auf Verträge, d.h. auf freiwillige Vereinbarungen angewiesen: Die dezentrale Verhandlungslösung tritt an die Stelle der zentralen Lösung (3). Solche Lösungen sind hier aber auch eher möglich, weil in der Regel die Zahl der beteiligten Akteure weitaus geringer ist als bei innerstaatlichen Maßnahmen; im Extremfall sind nur wenige oder gar nur zwei Staaten betroffen. Je geringer aber die Zahl der Beteiligten ist, desto geringer sind - ceteris paribus - die Anreize für Trittbrettfahrerverhalten und desto eher kann man zu freiwilligen Vereinbarungen kommen.

Solche Vereinbarungen setzen im allgemeinen voraus, daß für alle Beteiligten die Nutzen höher sind als die entstehenden Kosten. Die besondere Schwierigkeit internationaler Umweltschutzabkommen ergibt sich daraus, daß Nutzen und Kosten häufig sehr ungleich verteilt sind: Neben Ländern, die netto von solchen Maßnahmen erheblichen Nutzen haben, gibt es Länder, die Nettoverluste aufweisen. Solche Länder werden sich an entsprechenden Abkommen nur dann beteiligen, wenn sie fuir die ihnen entstehenden Kosten (weitgehend) kompensiert werden. Dies aber hat zur Konsequenz, daß im internationalen Bereich häufig der 'Verursacher' entschädigt wer- den muß, wenn er die Verschmutzung unterlassen soll. Ein wesentliches politisches Problem besteht daher in der politischen Durchsetzbarkeit von Abkommen mit Kompensationszahlungen.

Unter zwei Bedingungen sind Kompensationszahlungen vergleichsweise leicht durchsetzbar. Zum einen dann, wenn solche Zahlungen 'moralisch' in die richtige Richtung gehen. Wenn z.B. Entwicklungsländer dafür entschädigt werden sollen, daß sie auf die Rodung des tropischen Regenwaldes verzichten, wird sich in den entwickelten Staaten nur wenig politischer Widerstand regen. Die zweite Bedingung besteht darin, daß Vertragsbündel geschnürt werden, wodurch die Kompensationszahlungen nach außen nicht eindeutig als solche erkennbar sind. Das Schnüren von Vertragsbiundeln hat zudem den Vorteil, daß dadurch sichergestellt werden kann, daß möglichst viele der betroffenen Länder ein Interesse am Zustandekommen eines Vertrags haben, welches über das Interesse am Erhalt von Kompensationszahlungen hinausgeht. Da diese Zahlungen teilweise umstritten und damit langfristig wenig glaubwürdig sind, erhöht die Verknüpfung verschiedener Verträge, bei denen unterschiedliche Staaten Netto-Nutznießer sind, die Stabilität solcher Verträge.

\section{Konsequenzen}

Für die (nationale) Politik zur Bewältigung globaler Umweltprobleme ergeben sich daraus folgende Konsequenzen:

- Soweit dies möglich ist, sind im Bereich globaler Umweltprobleme internationale Abkommen anzustreben. Die unterschiedlichen Erfahrungen mit den Reduktionen des Ausstoßes von FCKW und Kohlendioxid zeigen jedoch, daß solche Abkommen nur unter bestimmten Bedingungen möglich sind. Daher ist zum einen nach Strategien zu suchen, um die internationale Akzeptanz solcher Abkommen zu erhöhen. Instrumente wie 'Dept for Nature Swaps' oder 'Joint Implementation' haben hier ihren Platz. Zweitens ist zu fragen, wie Politiken, welche einen Beitrag zur Bewältigung internationaler Umweltprobleme leisten, auch nationale Akzeptanz finden können. Dies wird dann am ehesten möglich sein, wenn sie auch einen Beitrag zur Verbesserung der nationalen Umweltsituation leisten.

- Damit die nationale Umweltpolitik im Prozeß der Globalisierung nicht 'unter die Räder' kommt, muß sie ökonomisch so effizient wie möglich durchgeführt werden. In diesem Zusammenhang kann neben Zertifikatslösungen 
eine ‘ökologische Steuerreform’ eine wesentliche Rolle spielen (4). Soweit durch eine solche Reform die bürokratische Umweltpolitik, wenn auch nicht ganz abgelöst, so doch in den Hintergrund gedrängt wird, führt sie zu Effizienzgewinnen. Da die Erhöhung der Produktionskosten durch die Verteuerung der Energie durch die Verringerung der Arbeitskosten zumindest teilweise kompensiert wird, ist eine solche Politik auch angesichts der wirtschaftlichen Globalisierung möglich.

Dies aber bedeutet, daß weder die wirtschaftliche Globalisierung nationale Umweltpolitik verunmöglicht, noch globale Umweltprobleme ohne politische Antwort bleiben müssen. In beiden Fällen wird die Umweltpolitik schwieriger und erfordert deshalb mehr (politische) Phantasie als zuvor, aber es stehen dennoch auch nationale umweltpolitische Handlungsmöglichkeiten zur Verfügung. Diese sollten gerade dann offensiv genutzt werden, wenn sich die Erzielung wirksamer internationaler Abkommen als schwierig erweist.

\section{Anmerkungen}

(1) Kirchgässner, G.: Probleme internationaler Umweltpolitik, in: E. Gawel (Hg.): Institutionelle Probleme der Umweltpolitik, Sonderheft 8/1996 der Zeitschrift für Angewandte Umwelfforschung, S.157-172.

(2) $\mathrm{Vgl}$. zu den Möglichkeiten eines kleinen Landes wie der Schweiz: Jochimsen, M., G.Kirchgüssner (Hg.): Schweizerische Umweltpolitik im internationalen Kontext, Birkhäuser, Bosel 1995.

(3) Vgl. z.B. Stieger, R.: Internationaler Umweltschutz: Eine politisch-ökonomische Analyse der Verträge zum Schutz der Ozonschicht, Peter Lang, Bern u.a. 1995.

(4) Zu Einzelheiten vgl. Kirchgässner, G.: Ökologische Steuerreform: Utopie oder realistische Alternative?, in: G. Krause-Junk (Hg.): Die Zukunft des Steuersystems, Sonderheft der Zeitschrift für Wirtschafts- und Sozialwissenschaften, Duncker und Humblot, Berlin 1998, S.279-319

\section{Der Autor}

Dr. Gebhard Kirchgässner ist Professor für Volkswirtschaftslehre und Ökonometrie sowie Direktor des Schweizerischen Instituts für AußenwirtschaftsStruktur- und Regionalforschung an der Universität Si. Gallen.

Kontakt: Universität Si. Gallen, SIASR, Institutsgebäude, Dufourstr. 48, CH-9000 St.Gallen. Tel: $++41 / 71 / 224-2347$.

E-mail: Gebhard.Kirchgaessner@SIASR.unisg.ch

Die europäische Integration im Vergleich mit globalen Entwicklungen

\title{
Liberalisierung und Umweltschutz
}

\author{
Im Zusammenhang mit der Globalisierung von Märkten wird vielfach die \\ Befürchtung eines umweltpolitischen "race to the bottom "geäußert. Der \\ europäische Fall zeigt jedoch, daß ökonomische Integration und Umweltschutz \\ keineswegs unvereinbare Ziele sein müssen, sofern eine geeignete Dynamik \\ supranationaler Kooperation entsteht.
}

$\mathrm{H}$ Von Christoph Knill äufig wird angenommen, daß bei zunehmender ökonomischer Interdependenz strenge Umweltstandards die nationale Industrie belasten und damit deren internationale Wettbewerbsfähigkeit unnötig schwächen würden. Vergleicht man dieses Szenario jedoch mit der Entwicklung der Umweltpolitik im Rahmen der Europäischen Union (EU), so scheint diese Prognose keineswegs universale Gültigkeit zu haben. Die Verwirklichung des europäischen Binnenmarktes hat nicht zu einem umweltpolitischen "race to the bottom" geführt. Vielmehr haben sich nationale Umweltregulierungen unter europäischem Einfluß tendenziell weiter verschärft (1). Es ist an dieser Stelle zu betonen, daß die Einschätzung der europäischen Umweltpolitik relativ zu globalen Entwicklungen erfolgt. Genauer betrachtet ist das Bild natürlich keineswegs so makellos, wie es sich aus dieser, ,Vogelperspektive" darstellt, nimmt man etwa die Vollzugsdefizite europäischer Umweltpolitik als Beispiel (2). Vor dem Hintergrund dieser relativ erfolgreichen Symbiose von Marktliberalisierung und Umweltschutz stellt sich die Frage, welche Bedingungen die europäische Entwicklung begünstigt haben und inwieweit diese Faktoren auch im Hinblick auf globale Umweltregulierung gegeben sind.

Betrachtet man die Entwicklung der europäischen Umweltpolitik, so fallen mehrere Aspekte auf, die auf den ersten Blick relativ überraschend sind - zumindest aus der Sicht der eingangs vorgestellten Annahme eines umweltpolitischen Dumpings. Erstens läßt sich eine graduelle Emanzipation der Umweltpolitik als eigenständiges Handlungsfeld der Gemeinschaft beobachten; d.h. Umweltpolitik wird nicht als Anhängsel, sondern losgelöst von Fragen der wirtschaftlichen Integration betrachtet (3). Diese Entwicklung wurde rechtlich bestätigt durch die vertragliche Verankerung von Umweltpolitik als eigenständiges Handlungsfeld der Gemeinschaft im Rahmen der Einheitlichen Europäischen Akte 1986 und deren Forfführung im Vertrag von Maastricht. Ein zweiter Aspekt, welcher die Entwicklung der europäischen Umweltpolitik charakterisiert, ist die relative, wenngleich unstetig verlaufende, Verschärfung umweltpolitischer Vorgaben. Diese bezieht sich nicht nur auf materielle Regulierungen (z.B. Emissionsgrenzwerte), sondern auch auf prozedurale Vorgaben (wie etwa den freien Zugang zu Umweltinformationen). Drittens ist schließlich die breite Mischung von verschiedenen Regulierungsmustern zu erwähnen. Europäische Umweltpolitik besteht keineswegs aus einem einheitlichen regulativen Konzept, sondern umfaßt eine Mischung von regulativen Ansätzen, welche von materiellen Standards und prozeduralen Vorgaben bis hin zur Selbstregulierung der Industrie reicht. Fragt man nach den Bedingungen, welche diese Entwicklung begünstigt haben, so lassen sich ökonomische und politische Mechanismen unterscheiden.

\section{Umwelidynamik des Binnenmarktes}

Wie David Vogel kürzlich gezeigt hat, muß ökonomische Integration keineswegs ein umweltpolitisches ,race to the bottom“ zur Folge haben, sondern kann unter bestimmten Bedingungen sogar ein „race to the top" begünstigen, d.h. eine Verschärfung von umweltpolitischen Regulierungen (4).

Einerseits ist es insbesondere in umweltpolitisch hochentwickelten Staaten denkbar, daß die Industrie für eine weitere Verschärfung nationaler Umweltstandards eintritt, um Wettbewerbsvorteile gegenüber ausländischen Konkurrenten zu erlangen. Dies ist dann der Fall, wenn die nationale Industrie bereits auf solche Standards einge- 
(c) 20I0 Authors; licensee IÖW and oekom verlag. This is an article distributed under the terms of the Creative Commons Attribution Non-Commercial No Derivates License (http://creativecommons.org/licenses/by-nc-nd/3.o/), which permits unrestricted use, distribution, and reproduction in any medium, provided the original work is properly cited. 